\title{
Discrimination of Isomeric Fragment Ions Observed in Tandem Mass Spectra of Biantennary Oligosaccharides by Use of Selective Isotope Labeling
}

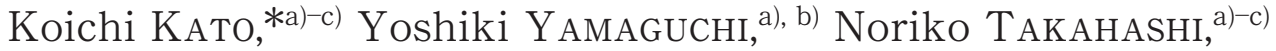 \\ Mamiko Nishimura, a), b) Shin-ichi Iwamoto, d) \\ Sadanori SeKiya, ${ }^{\text {d) }}$ and Koichi TANAKA ${ }^{\text {d) }}$
}

(Received May 26, 2004; Accepted June 16, 2004)

\begin{abstract}
We report herein the first mass spectrometric analyses of isotopomers of a pyridylamino (PA)-derivatized biantennary oligosaccharide, in which one of two non-reducing terminal galactose residues is selectively enriched in ${ }^{13} \mathrm{C}$ by an enzymatic reaction. By use of these isotopomeric PA-oligosaccharides, individual fragment ions observed in tandem mass spectra of MALDI-QIT-TOF-MS were unambiguously assigned. It was shown that the oligosaccharide ion that lacks the $N$-acetyllactosamine previously linked to the Man $\alpha 1 \rightarrow 6$ antenna dominates its isomeric counterpart ion. We propose that tandem mass spectrometric analyses of oligosaccharides isotopically labeled at a selected antenna would provide us with the basis of mechanisms of formation of glycosidic linkage fragment ions.
\end{abstract}

\section{Introduction}

Carbohydrate chains carry a variety of biological functions, e.g., mediating cell-cell communication and contributing to folding and quality control of proteins in cells. ${ }^{1-4)}$ For detailed understanding of mechanisms of glycoconjugate functions, it is an essential step to determine structures of their carbohydrate chains.

Mass spectrometry (MS) is becoming the method of choice for rapid and convenient analyses of oligosaccharide structures. It is, however, not feasible to determine an oligosaccharide sequence unambiguously solely on the basis of MS data because there exist a variety of isomeric structures with different composition, glycosidic linkage, and branching from one another. Recently, evidence is accumulating that abundances of glycosidic linkage fragment ions, which are observed in tandem mass spectrometric and postsource decay (PSD) fragment analyses, are dependent on composition, ${ }^{5), 6)}$ glycosidic linkage, ${ }^{6-10)}$ and branching. ${ }^{11), 12)}$ This suggests that oligosaccharide sequencing discriminating isomeric structures can be deduced from the analyses of relative abundance of fragment ions. However, identification of individual fragment ions originating from the multiantennary oligosaccharides is generally quite difficult. Hence little has been known about mechanisms of preferential cleavages of specific glycosidic linkages and formation of fragment

*a) Graduate School of Pharmaceutical Sciences, Nagoya City University (3-1 Tanabe-dori, Mizuho-ku, Nagoya 4678603, Japan

b) CREST, JST (4-1-8 Honcho, Kawaguchi 332-1102, Japan)

c) GLYENCE Co., Ltd. (1-4-6 Masaki, Naka-ku, Nagoya 460-8690, Japan)

d) Koichi Tanaka Mass Spectrometry Research Laboratory, Shimadzu Corporation (1 Nishinokyo-Kuwabaracho, Nakagyo-ku, Kyoto 604-8511, Japan) ions.

We report herein the first mass spectrometric analyses of isotopomers of a pyridylamino (PA)-derivatized biantennary oligosaccharide, in which one of two nonreducing terminal galactose (Gal) residues is selectively enriched in ${ }^{13} \mathrm{C}$. By use of these isotopomeric PAoligosaccharides, individual fragment ions observed in tandem mass spectra of MALDI-QIT-TOF-MS were unambiguously assigned.

\section{Experimental}

Biantennary oligosaccharides 1,2 , and 3 were liberated by a glycoamidase A treatment from the glycopeptides of human immunoglobulin G1, and pyridylaminated giving rise to $\mathrm{PA}$-derivatives $\mathbf{4 a}, \mathbf{5 a}$, and $\mathbf{6 a}$ (Fig. 1) respectively, which were then separated on an ODS reverse-phase HPLC column with a linear gradient of butanol in sodium phosphate buffer. ${ }^{13)}$ The PAderivatization is crucial to separating those isomeric oligosaccharides. ${ }^{14)}$ For the preparation of PA-derivatized oligosaccharide isotopomers $6 \mathbf{b}$ and $\mathbf{6 c}$, in vitro enzymatic galactosylation ${ }^{15)}$ was used as the key reaction (Fig. 2). By successive one pot enzymatic reactions, D- $\left[\mathrm{U}_{-}{ }^{13} \mathrm{C}_{6}\right] \mathrm{Gal}$ was phosphorylated, ${ }^{16)}$ converted to UDP-[U- $\left.{ }^{13} \mathrm{C}_{6}\right] \mathrm{Gal},{ }^{17),}{ }^{18)}$ and then attached onto the non-reducing terminal $N$-acetylglucosamine (GlcNAc) residue of $4 \mathrm{a}$ and $5 \mathrm{a}$, giving rise to $6 \mathrm{~b}$ and $6 \mathrm{c}$, respectively. Three isotopomers of the digalactosyl PA-oligosaccharide, $\mathbf{6 a}, \mathbf{6 b}$, and $\mathbf{6 c}$ were obtained and these samples were subjected to MALDI-QIT-TOF-MS measurements using recrystallized 2,5-dihydroxybenzoic acid as matrix.

\section{Results and Discussion}

In mass spectrum of $6 \mathbf{a}, 6 \mathbf{b}$, and $6 \mathbf{c}$, respectively, sodium adducted molecular ion was detected as a main peak. In the tandem mass spectrum of $[6 \mathbf{a}+\mathrm{Na}]^{+}$(theo- 


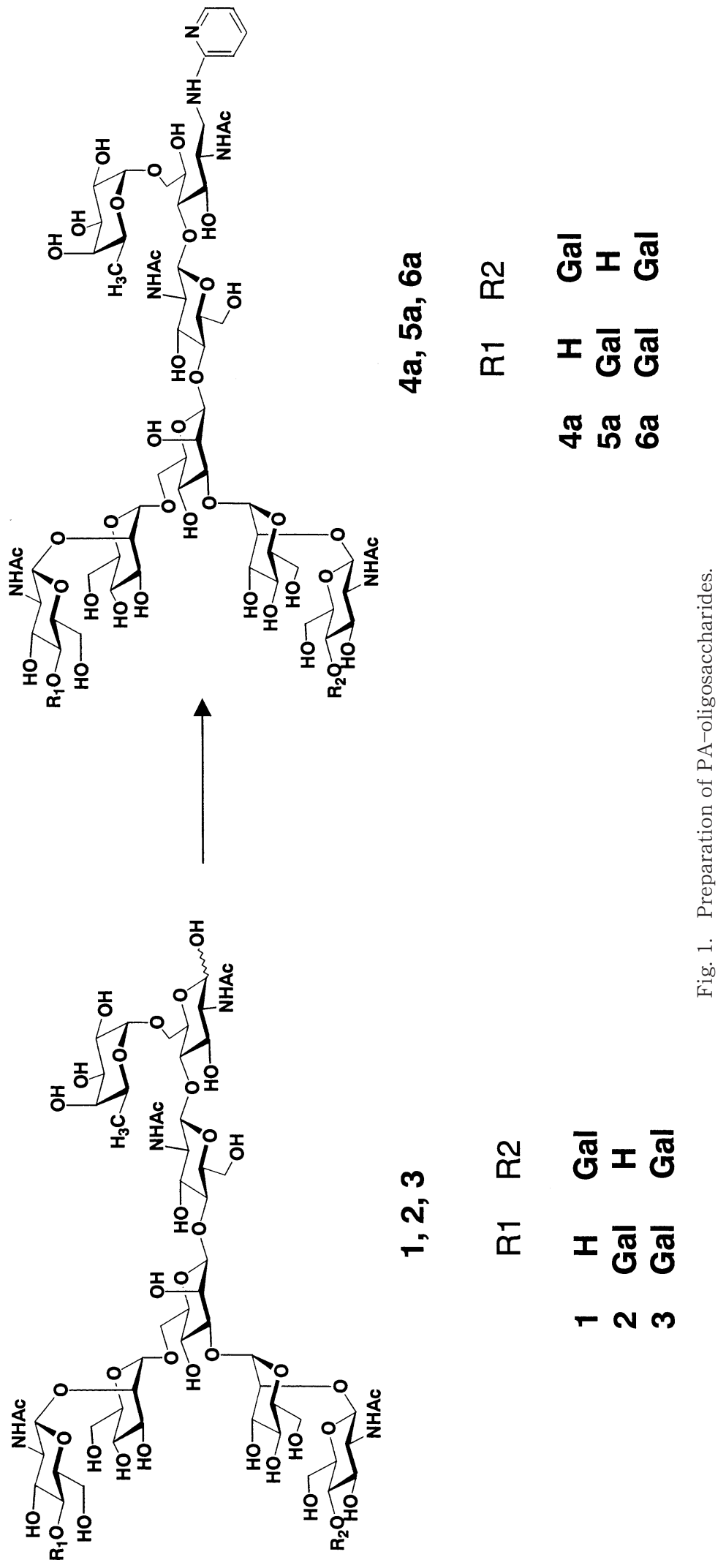




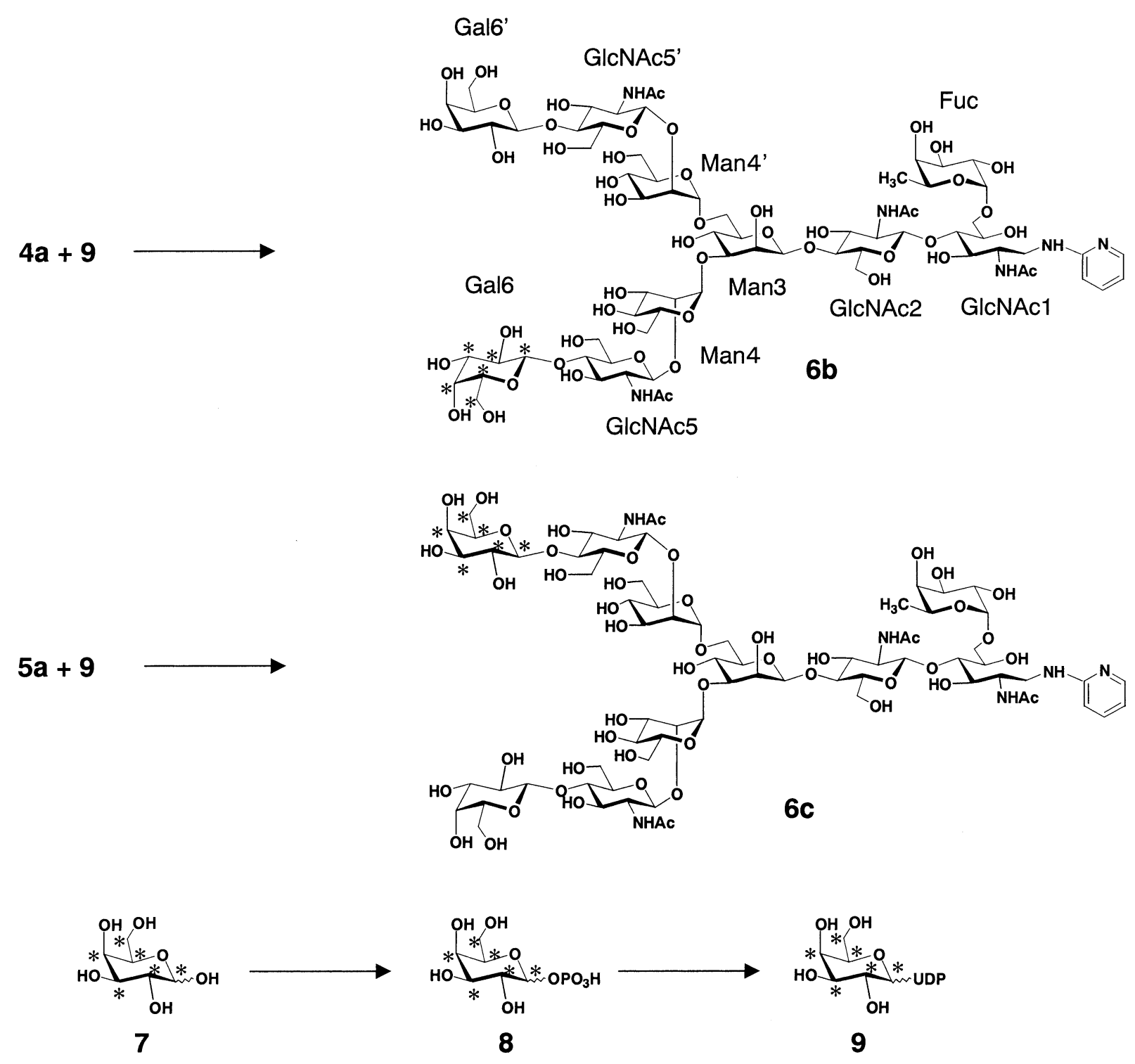

Fig. 2. Preparation of ${ }^{13} \mathrm{C}$-labeled $\mathrm{PA}$-oligosaccharides.

retical monoisotopic molecular mass: 1887.70), glycosidic linkage fragment ions were observed at $\mathrm{m} / \mathrm{z}$ 1522.66, 1442.65, 1077.46, and 874.37 (Fig. 3A). These fragment ions indicates that cleavages occur at the GlcNAc $\beta 1 \rightarrow 2$ mannose (Man), Man $\beta 1 \rightarrow 4 \mathrm{GlcNAc}$, and GlcNAc $\beta 1 \rightarrow 4 \mathrm{GlcNAc}$ glycosidic linkages, while cleavage at the Gal $\beta 1 \rightarrow 4$ GlcNAc glycosidic linkage is negligible. The fragment ion at $m / z 1522.66$ corresponds to elimination of $N$-acetyllactosamine from one of the two antennae of $\mathbf{6 a}$. However, it is not possible to tell which antenna lost the $N$-acetyllactosamine based on this result. On the other hand, in the tandem mass spectra of $[6 \mathbf{b}+\mathrm{Na}]^{+}$(theoretical molecular mass: 1893.72), elimination of the $N$-acetyllactosamine gave rise to pair fragment ions with the mass difference of $6 \mathrm{Da}$, which correspond to at $m / z 1522.77$ and $m / z 1528.78$ (Figs. 3 $\mathrm{B}$ and $\mathrm{F}$ ). The intensity ratio of the peak at $\mathrm{m} / \mathrm{z}$ 1522.77 to the peak at $m / z 1528.78$ was $c a .2: 5$, indicating that the oligosaccharide ion that lacks the $N$ acetyllactosamine previously linked to the Man $\alpha 1 \rightarrow 6$ antenna dominates its isomeric counterpart ion. A similar tendency was observed in the other pair fragment ions with the mass difference of 6 Da (Figs. 3D and E). Consistently, relative peak intensities were reversal for the pair peaks observed in the tandem mass spectra of $[\mathbf{6 c}+\mathrm{Na}]^{+}$(Figs. $3 \mathrm{C}, \mathrm{G}, \mathrm{H}$, and I).

The different incidence between the two isomeric fragment ions might be attributed to a preferential cleavage of the GlcNAc $\beta 1 \rightarrow 2$ Man glycosidic linkage in the Man $\alpha 1 \rightarrow 6$ antenna. An alternative explanation is that relative $\mathrm{Na}^{+}$-binding affinities are different between the two antennae where the $N$-acetyl groups of the GlcNAc residues form major binding-sites of alkali metal ions. ${ }^{19)}$ Presupposition of the above two hypotheses is that chemical properties of the two GlcNAc residues, i.e., GlcNAc5 and GlcNAc5', are considerably different with each other. The third explanation is that efficiency of $\mathrm{Na}^{+}$transfer upon fragmentation from the GlcNAc residue (GlcNAc5 or GlcNAc5'), which is being eliminated, to the remaining GlcNAc2 is different between the two antennae, which results in different incidence of observed fragment ions, even if there is no significant difference in $\mathrm{Na}^{+}$-binding affinities among the GlcNAc residues. It has been shown that GlcNAc5' can be closer to GlcNAc2 than GlcNAc5 is, since the Man $\alpha 1 \rightarrow 6$ Man glycosidic linkage is more flexible than 


\begin{tabular}{lll|}
\hline Galactose $\left({ }^{12} \mathrm{C}\right)$ & $\bigcirc$ Mannose & $\triangle$ Fucose \\
$\star$ Galactose $\left({ }^{13} \mathrm{C}\right)$ & $\square$ GlcNAc & 凸 Pyridylamino \\
\hline
\end{tabular}
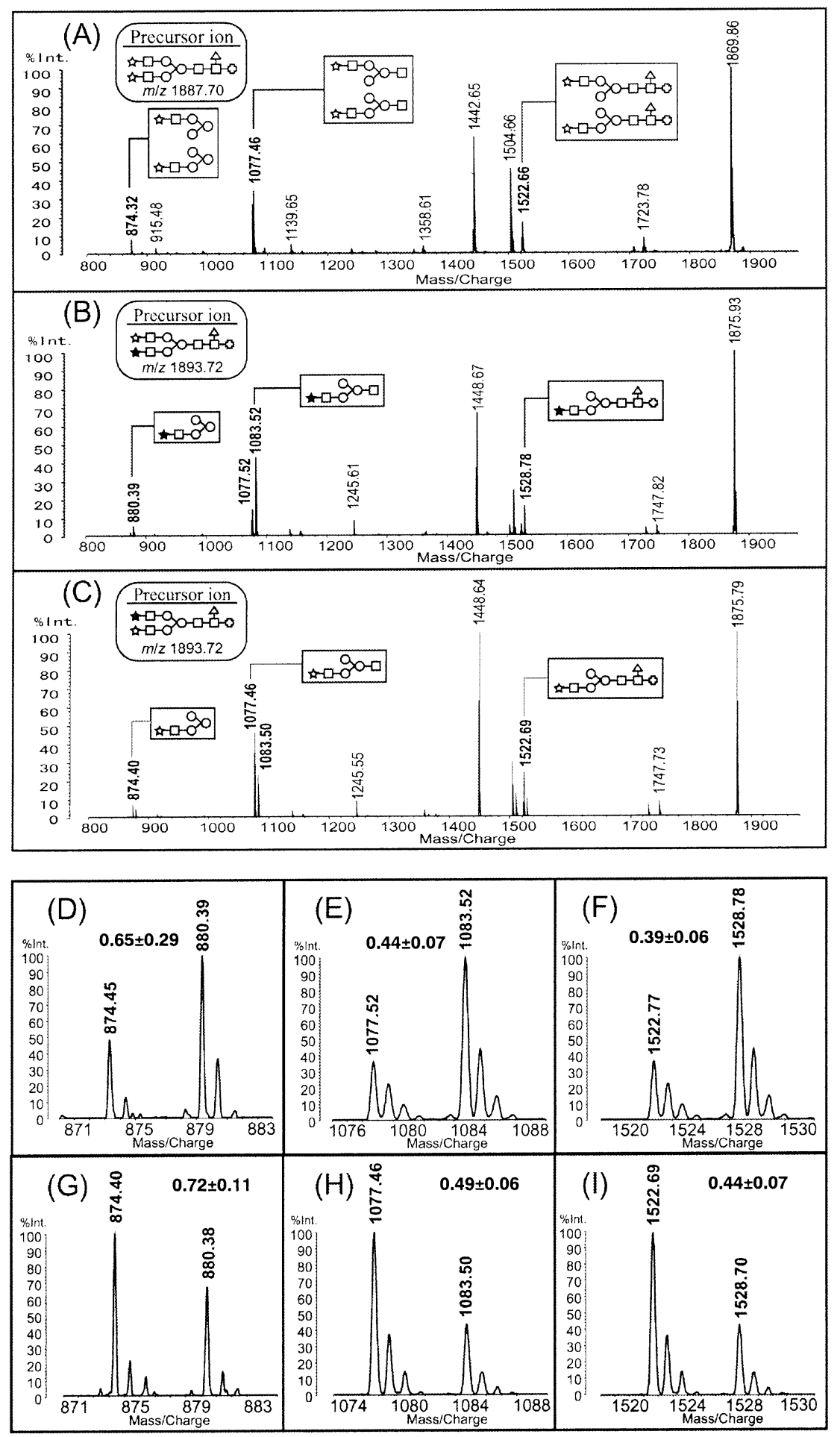

Fig. 3. The tandem mass spectra of sodium adducted PA-oligosaccharide with MALDI-QIT-TOF-MS. (A) 6a. (B) $6 \mathbf{b}$ (C) 6c. (D), (E), and (F) The magnification of three pairs of peaks observed in the tandem mass spectrum of 6b. (G), (H), and (I) The magnifications of three pairs of peaks observed in the tandem mass spectrum of $6 \mathbf{c}$. The intensity ratios of the minor peak to the major peak (the average and standard deviation for eight measurements) are indicated in D to I.

that of the Man $\alpha 1 \rightarrow 3$ Man glycosidic linkage. ${ }^{20), 21)}$ Therefore it is possible that elimination of Gal6'GlcNAc5' gives rise to observable fragment ion more efficiently than that of Gal6-GlcNAc5.

\section{Conclusions}

In conclusion, structure of isomeric fragment ions observed in the tandem mass spectrum could be unam- 
biguously assigned by use of oligosaccharide isotopomers in which selected positions are enriched in ${ }^{13} \mathrm{C}$. The data obtained by use of this technique would provide us with the basis of mechanisms of formation of glycosidic linkage fragment ions. A more systematic study is under way in our laboratories.

\section{Acknowledgement}

This work was supported in part by Grants-in-Aid from the Ministry of Education, Culture, Sports, Science and Technology of Japan.

\section{References}

1) R. A. Dwek, Chem. Rev., 96, 683 (1996).

2) A. Varki, Glycobiology, 3, 97 (1993).

3) H. Lis and N. Sharon, Eur. J. Biochem., 218, 1 (1993).

4) A. Kobata, Eur. J. Biochem., 209, 483 (1992).

5) T. Yamagaki and H. Nakanishi, Rapid Commun. Mass Spectrom., 12, 1069 (1998).

6) T. Yamagaki and H. Nakanishi, Proteomics, 1, 329 (2001).

7) Y. Sato, M. Suzuki, T. Nirasawa, A. Suzuki, and T. Endo, Anal. Chem., 72, 1207 (2000).

8) T. Yamagaki and H. Nakanishi, Anal. Sci., 17, 83 (2001).

9) T. Yamagaki and H. Nakanishi, J. Mass Spectrom., 35, 1300 (2000).

10) T. Yamagaki and H. Nakanishi, Glycoconj. J., 16, 385 (1999).
11) H. L. Cheng and G. R. Her, J. Am. Soc. Mass Spectrom., 13, 1322 (2002).

12) Y. Takegawa, S. Ito, S. Yoshioka, K. Deguchi, H. Nakagawa, K. Monde, and Nishimura, Rapid Commun. Mass Spectrom., 18, 385 (2003).

13) M. Holland, K. Takada, T. Okumoto, N. Takahashi, K. Kato, D. Adu, A. Ben-Smith, L. Harper, C. O. Savage, and R. Jefferis, Clin. Exp. Immunol., 129, 183 (2002).

14) F. H. Routier, E. F. Hounsell, P. M. Rudd, N. Takahashi, A. Bond, F. C. Hay, A. Alavi, J. S. Axford, and R. Jefferis, J. Immunol. Methods, 213, 113 (1998)

15) C.-H. Wong, R. Wang, and Y. Ichikawa, J. Org. Chem., 57, 4343 (1992).

16) J. E. Heidas, W. J. Lees, and G. M. Whitesides, J. Org. Chem., 57, 152 (1992).

17) H. M. Kalckar, B. Braganca, and A. Munch-Petersen, Nature, 172, 1038 (1953).

18) K. Kurahashi and A. Sugimura, J. Biol. Chem., 235, 940 (1960).

19) M. T. Cancilla, A. W. Wong, L. R. Voss, and C. B. Lebrilla, Anal. Chem., 71, 3206 (1999).

20) S. W. Homans, R. A. Dwek, D. L. Fernandes, and T. W. Rademacher, FEBS Lett., 150, 503 (1982).

21) H. J. Stubbs, J. J. Lih, T. L. Gustafson, and K. G. Rice, Biochemistry, 35, 937 (1996).

Keywords: Oligosaccharides, Tandem mass spectra, Isotope, Isomers, Ion trap quadrupole 